Methods 427 subjects with a wide range of health and disease were divided into derivation $(\mathrm{n}=214)$ and validation $(\mathrm{n}=213)$ cohorts (Table 1 for patient characteristics). All subjects underwent T1 mapping with ShMOLLI at 1.5 Tesla for ECV quantification. Venous blood for Hct was obtained prior to scanning with 44 patients having a repeat Hct within $6 \mathrm{~h}$.

$\mathrm{ECV}$ was calculated as: $\mathrm{ECV}=\left(\Delta\left[1 / \mathrm{T} 1_{\text {myo }}\right] / \Delta\left[1 / \mathrm{T} 1_{\text {blood }}\right]\right) *$ [1-haematocrit]).

Synthetic Hct was approximated from the linear relationship between Hct and native $\mathrm{T} 1_{\text {blood }}$, and used to calculate synthetic ECV. Histological validation was performed on 18 patients with severe aortic stenosis (age $71 \pm 10$ years, $78 \%$ male). ECV was compared with collagen volume fraction from intra-operative biopsies taken during surgical valve replacement.

Results In the derivation cohort, native $\mathrm{T} 1_{\text {blood }}$ and Hct showed a linear relationship $\left(\mathrm{R}^{2}=0.45 ; p<0.001\right.$, Figure 1$)$. This was used to derive synthetic Hct $=0.88-\left(\mathrm{T} 1_{\text {blood }} /\right.$ 3240). Synthetic ECVcorrelated well with ECV $\left(\mathrm{R}^{2}=0.99\right.$; $p<0.001)$. These results were maintained in the validation cohort. Test:retest variability of haematocrit was higher than expected ( $n=44$, variability $10 \%$ with Hct:Hct $R^{2}=0.86$ ). On histological validation, synthetic and conventional ECV both correlated well with collagen volume fraction $\left(\mathrm{R}^{2}=0.61\right.$ and $0.69, p<0.001)$.

Conclusion Synthetic ECV allows instantaneous non-invasive quantification of the myocardial extracellular space without blood sampling. Inline application of synthetic ECV may be an attractive alternative in clinical practice.

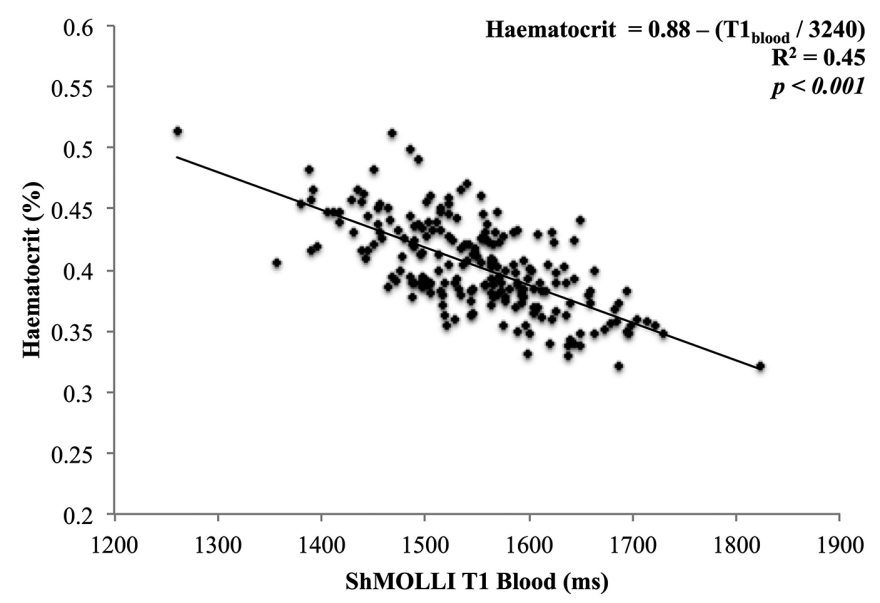

Abstract 29 Figure 1 Correlation between T1 blood and haematocrit. In the derivation cohort $(\mathrm{n}=214)$, native $\mathrm{T1}$ blood and hematocrit $(\mathrm{Hct})$ showed a linear relationship ( $R 2=0.45 ; p<0.001)$. This was used to derive synthetic $\mathrm{Hct}=0.88-\left(\mathrm{T} 1_{\text {blood }} / 3240\right)$.

\section{WHOLE BODY CONTRAST ENHANCED MRA CAN QUANTIFY AND MONITOR ATHEROSCLEROSIS PROGRESSION}

${ }^{1} \mathrm{JR}$ Weir-McCall, ${ }^{2} \mathrm{RD}$ White, ${ }^{3} \mathrm{~S} J$ Gandy, ${ }^{4} \mathrm{PG}$ Ramkumar, ${ }^{1} \mathrm{JJF}$ Belch, ${ }^{1} \mathrm{AD}$ Struthers, $1,4 \mathrm{JG}$ Houston. ${ }^{1}$ Division of Cardiovascular and Diabetes Medicine, Medical Research Institute, University of Dundee, DD1 9SY, UK; ${ }^{2}$ Department of Clinical Radiology, University Hospital of Wales, Cardiff, CF14 4XW, UK; ${ }^{3}$ Medical Physics, Ninewells Hospital, Dundee, DD1 9SY, UK; ${ }^{4}$ Clinical Radiology, Ninewells Hospital, Dundee, DD1 9SY, UK

10.1136/heartjnl-2015-307845.30
Aim To determine the ability of whole body magnetic resonance angiography (WB-MRA) to measure global atheroma burden progression.

Methods 50 consecutive patients with symptomatic peripheral arterial disease referred for clinical MRA were recruited. WB-MRA was performed at baseline, 6 months and 3 years. WB-MRA data was analysed by dividing the vasculature into 31 anatomical arterial segments. Each segment was scored according to degree of luminal narrowing: $0=$ normal, $1=<50 \%, 2=50-70 \%, 3=71-99 \%, 4=$ vessel occlusion. From this a standardised atheroma score (SAS) was calculated with a maximum score of 100 and minimum score of 0 . Progression was assessed with repeat measure ANOVA.

Results 36 patients were scanned at 0 and 6 months, with 26 patients scanned at the three year follow up. Only those who completed all 3 visits were included in the final analysis. At 3 years, $\mathrm{n}=18$ demonstrated atheroma progression while $\mathrm{n}=8$ showed stable or improved disease. Those with no progression had significantly lower baseline SAS, and were more likely to be on statin therapy ( $p<0.05$ for both). Baseline SAS was $15.7 \pm 10.3$ at baseline with no progression at 6 months $(\mathrm{SAS}=16.4 \pm 10.5, \mathrm{p}=0.67)$. At 3 years there was significant progression in atheroma $(\mathrm{SAS}=17.7 \pm 11.5, \mathrm{p}=0.01)$ (Figure 1). On multiple linear regression, age ( $\beta \quad 0.14$ $\mathrm{p}=0.014)$, pulse pressure $(\beta-0.12 \mathrm{p}=0.005)$ and anklebrachial pressure index $(\beta-7.7 \mathrm{p}=0.036)$ were independently associated with the rate of progression.

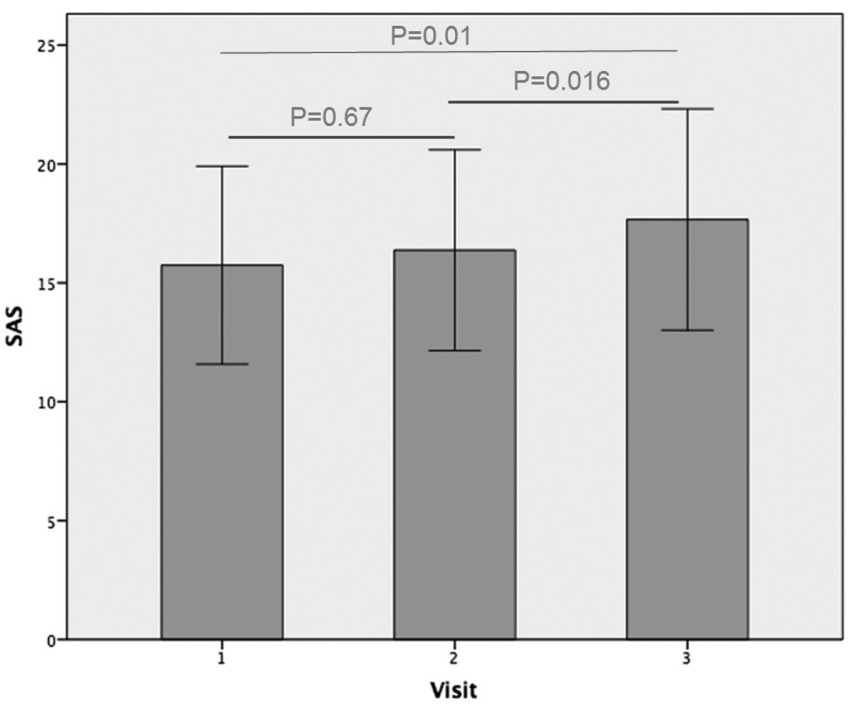

Abstract 30 Figure 1 Comparison of atheroma score at baseline, 6 months and 3 years. Visit $1=$ Baseline, Visit $2=6$ months, Visit $3=$ 3 years. T-bars represent $95 \%$ confidence intervals.

Conclusion Whole body contrast enhanced MRA can quantify and monitor atherosclerosis progression at 3 year follow-up even in a small cohort.

\section{ISCHAEMIA AND VIABILITY ASSESSMENT WITH ADENOSINE STRESS CMR IN HIGH RISK PATIENTS: SAFETY, FEASIBILITY AND TOLERABILITY}

Catherine Wilson, Alexander Carpenter, Amardeep Ghosh Dastidar, Jonathan Rodrigues, Nauman Ahmed, Anna Baritussio, Chris Benny Lawton, Elisa McAlindon, Chiara BucciarelliDucci. NIHR Bristol Cardiovascular Biomedical Research Unit, Bristol Heart Institute, Bristol, UK 
Background Adenosine stress cardiovascular magnetic resonance (CMR) provides effective cardiac prognostication in patients with suspected coronary artery disease. However its use has been limited in high-risk patients and some reservations exist about offering adenosine stress CMR in patients with significant aortic stenosis, asthma, severe left ventricular (LV) systolic dysfunction, significant left main stem (LMS) disease and age $>80$ years.

Aims To determine the safety, tolerability and feasibility of adenosine stress CMR, in high-risk individuals.

Method Consecutive adenosine stress-CMR examinations (March 2013-March 2014) were included. A comprehensive adenosine stress CMR protocol was used. High-risk if: age $>80$ years, asthma, significant LMS stenosis and moderate-severe or severe aortic stenosis or severe LV systolic dysfunction (ejection fraction $<40 \%$ ). Fisher exact test for binary variables was used.

Results 574 consecutive stress-CMR were included in the analysis (mean age 64 years and $71 \%$ males), out of which 128 patients $(22 \%)$ met the criteria for high-risk. Overall, the complete stress-CMR protocol was successfully performed in 93\% of patients: the high-risk group had a drop out rate of $2 \%$ compared to a rate of $7 \%$ for the non-high risk group $(\mathrm{p}=0.08)$. Adequate stress response (symptomatic and/or cardiovascular) was achieved in 98\% of high-risk and $97 \%$ of non-high risk $(\mathrm{p}=0.55) .70 \%$ of high-risk patients received incremental increases in adenosine dose. Overall, no serious adverse events were noted. When compared to non-high risk, the high-risk group more commonly presented with inducible perfusion defects $(62 \%$ vs $42 \%, \mathrm{p}=0.0003)$ and LGE $(74 \%$ vs $55 \%, \mathrm{p}=0.0006)$.

Conclusion Adenosine stress-CMR is a safe, well-tolerated and feasible investigational modality even in high-risk individuals (moderate/severe aortic stenosis, significant LMS stenosis, severe LV systolic dysfunction, asthma/COPD or age $>80$ ) with known or suspected ischaemic heart disease. The incidence of myocardial ischemia or LGE is significantly higher in the high-risk group. 